\title{
Susceptibilidad in vitro a anidulafungina en 100 cepas de especies de Candida aisladas previo a la introducción de esta equinocandina en Chile
}

\author{
Claudio Alburquenque O., Viviana Silva A., Milenko Fuentes G., Cecilia Tapia C. y Víctor Silva V.
}

\section{In vitro andulafungin susceptibility of 100 of Candida strains obtained previously to the introduction of this echinocandin in Chile}

Fungi, particularly yeasts, have become important opportunistic pathogens that can be resistant to antifungal agents or develop resistance. To address this problem, new molecules such as echinocandins, have been developed. Susceptibility to anidulafungin was studied in yeasts isolated previous to the introduction of this drug in Chile. One hundred strains of different yeast species isolated from invasive fungal infections during 2007 and 2008 were studied. Susceptibility testing of anidulafungin was performed by broth microdilution according to CLSI. All strains were susceptible to anidulafungin. $\mathrm{MIC}_{50}$ and $\mathrm{MIC}_{90}$ were $0.125 \mu \mathrm{g} / \mathrm{mL}$ and $1 \mu \mathrm{g} / \mathrm{mL}$, respectively. Compared to other yeasts, C. parapsilosis showed a slight increase in the MICs for anidulafungin ( $\mathrm{MIC}_{50}$, $\left.1 \mu \mathrm{g} / \mathrm{mL}, \mathrm{MIC}_{90}, 2 \mu \mathrm{g} / \mathrm{mL}\right)$, but remained within the susceptible range. Both, fluconazole resistant (8) and dose dependant susceptible strains (16) were susceptible to anidulafungin. In vitro, this echinocandin appears to be an effective therapeutic alternative.

Key words: Antifungal susceptibility, yeasts, anidulafungin, equinocandin.

Palabras clave: Susceptibilidad antifúngica, levaduras, anidulafungina, equinocandina.

\section{Introducción}

$\mathrm{D}$ esde hace tres décadas los hongos han emergido como importantes agentes de infecciones oportunistas, que afectan especialmente a pacientes inmunocomprometidos ${ }^{1,2}$. El incremento de las enfermedades fúngicas invasoras (EFI) se ha mantenido constante en el ambiente hospitalario, debido fundamentalmente a un mayor número de pacientes con patologías de riesgo, tales como leucemias, cáncer con neutropenia inducida, pacientes con trasplante de precursores hematopoyéticos sometidos a terapias inmunosupresoras y pacientes con SIDA, entre otros ${ }^{3}$. Dentro de los agentes productores de micosis en este tipo de pacientes, la especie más frecuentemente aislada es Candida albicans; sin embargo, otras especies como C. glabrata, C. parapsilosis, C. tropicalis y C. krusei se han descrito como agentes causales de candidiasis en distinta frecuencia, siendo algunas de ellas resistentes a algunos antifúngicos ${ }^{2,4}$. En este contexto, se ha descrito la presencia de resistencia secundaria en cepas de Candida expuestas a fármacos fungistáticos como los azoles y el aislamiento de especies intrínsecamente resistentes a determinados antifúngicos como C. krusei $i^{5,6}$.

En Chile, estudios de vigilancia han descrito un aumento de aislamiento de especies de Candida no-albicans en infecciones fúngicas en general ${ }^{7-9}$. Estos datos concuerdan con otros estudios de vigilancia en Norteamérica y Latinoamérica, donde se ha observado que las especies de C. no-albicans, presentan cambios epidemiológicos continuos en su frecuencia con una tendencia al aislamiento de especies más resistentes a los antifúngicos ${ }^{2,10}$.

Para poder superar esta situación, en los últimos años se han desarrollado agentes antifúngicos de la clase equinocandinas, los cuales actúan por inhibición de la síntesis de 1,3- $\beta$-D-glucano en la pared celular fúngica ${ }^{11,12}$. Los antifúngicos de esta clase, como anidulafungina, caspofungina y micafungina, poseen actividad contra muchas especies del género Candida, incluyendo cepas resistentes a polienos y a azoles, transformándose en una excelente alternativa terapéutica frente a cepas resistentes a otros antimicóticos ${ }^{13-15}$. Los integrantes de esta familia de antifúngicos tienen una alta actividad in vitro asociada con una baja toxicidad en el tratamiento de infecciones graves por Candida ${ }^{16,17}$. Entre estos fármacos, anidulafungina ha sido aprobada en Estados Unidos de América por la FDA (Food and Drug Administration) en el año 2006 para el tratamiento de la candidiasis invasora, incluyendo candidemia $^{18}$.

En el presente trabajo se evaluó por primera vez en Chile la susceptibilidad in vitro a anidulafungina en
Universidad Mayor, Santiago, Chile. Facultad de Medicina (CAO, VSA, MFG, VSV).

Universidad de Chile, Santiago, Chile. Facultad de Medicina (CTC).

Los autores declaran no tener conflictos de interés. Trabajo Financiado con aportes de la Universidad Mayor.

Recibido: 20 de enero de 2011 Aceptado: 20 de mayo de 2011

Correspondencia a: Víctor Silva V.

victor.silva@umayor.cl. 
distintas especies de levaduras aisladas de pacientes con diagnóstico de EFI entre 2007 y 2008, previo a la incorporación de este fármaco en nuestro medio.

\section{Material y Métodos}

Cepas de levaduras. Se estudiaron 100 aislados, representativos de 100 pacientes entre los años 2007 y 2008 del programa IFI (Infecciones Fúngicas Invasoras)* de la Universidad Mayor/ Universidad de Chile y el Instituto de Salud Pública de Chile (ISP). Buscando una representatividad en la diversidad de especies de levaduras aisladas en nuestro medio, se procedió a seleccionar aislados de Candida albicans (n: 33), C. parapsilosis (n: 26), C. glabrata (n: 19), C. tropicalis (n: 9), C. lusitaniae (n: 5), C. krusei (n:4), C. guilliermondii (n: 2), C. rugosa (n: 1) y Saccharomyces cerevisiae (n: 1), elegidos al azar entre las cepas de cada una de las respectivas especies mantenidas en el cepario, independientes de su patrón de sensibilidad previa. Se recolectaron los principales antecedentes demográficos de los pacientes registrados en fichas ad-hoc, como tipo de muestra, edad de los pacientes, considerando niños a pacientes hasta 15 años de edad. Las cepas fueron recuperadas de su crio-conservación en leche descremada al $20 \%$ a $-20^{\circ} \mathrm{C}$. Cada cepa fue repicada en agar Sabouraud glucosado y medio cromogénico (ChromAgar Candida ${ }^{\circledR}$ ), siendo incubadas entre 24 y $48 \mathrm{hrs} \mathrm{a} 37^{\circ} \mathrm{C}$. Esta siembra se repitió tres veces antes de realizar los estudios, para garantizar el crecimiento exponencial en la mayoría de los individuos del cultivo.

Identificación de levaduras. Las especies de levaduras se identificaron según procedimientos estándares empleados en micología médica, analizando las características fisiológicas, morfológicas y bioquímicas de las levaduras, empleando prueba de tubo germinal en plasma fresco humano, microcultivo en agar maíz con tween 80 al 1\% y galerías API 32C (Biomerieux), respectivamente ${ }^{7,19,20}$.

Medición de la concentración inhibitoria mínima. Las 100 cepas incorporadas, presentaban estudio previo de susceptibilidad in vitro frente a anfotericina B, fluconazol, voriconazol y caspofungina mediante método de difusión por discos (Neosensitab, ROSCO $®)$ y/o E-test $\AA^{21-25}$. Las cepas fueron sembradas en agar Sabouraud glucosado e incubadas por $18 \mathrm{hrs}$ antes de los ensayos. La susceptibilidad a anidulafungina fue determinada por microdilución en caldo según el documento M27-A2 de la CLSI (Clinical Laboratory Standards Institute) ${ }^{26}$, utilizando como control las cepas C. krusei ATCC 6258 y C. parapsilosis ATCC $22019^{27}$. En resumen, anidulafungina (ECALTA $®$ Pfizer) se obtuvo en su presentación original en polvo, diluyendo el fármaco en dimetil sulfóxido (DMSO), obteniendo una concentración de 1,6 mg/mL. Luego se realizaron diluciones en RPMI 1640 con L-glutamina y tamponado con MOPS (Sigma Aldrich ${ }^{\circledR}$ ), para finalmente cargar la microplaca con $100 \mu \mathrm{L}$ de las distintas concentraciones entre 32 $\mu \mathrm{g} / \mathrm{mL}$ a $0,0625 \mu \mathrm{g} / \mathrm{mL}$. Las levaduras en estudio fueron preparadas en agua destilada estéril a una concentración inicial de $1-5 \times 10^{6} \mathrm{ufc} / \mathrm{mL}$ posteriormente diluidas 1:50, para finalmente diluirlas en RPMI 1640 con L-glutamina y MOPS (1:20). Luego se agregó $100 \mu \mathrm{L}$ del inóculo en las microplacas que contenían las distintas concentraciones del fármaco. La concentración final de las levaduras en cada pocillo fue de $0,5-2,5 \times 10^{3} \mathrm{ufc} / \mathrm{mL}$. Como control de crecimiento se utilizó el solvente más inóculo en el caldo de cultivo y el control negativo fue agua destilada. Consideramos sensibilidad a anidulafungina si la cepa presentó valores $\mathrm{CIM} \leq 2 \mu \mathrm{g} / \mathrm{mL}^{17}$.

\section{Resultados}

Los principales datos de los pacientes asociados a las cepas seleccionadas son predominio de la especie $C$. glabrata en adultos con 18 aislados en comparación con una cepa recuperada de niño. Por otra parte, las cepas de C. parapsilosis correspondieron principalmente a aislados de niños (n: 17) respecto de los adultos (n: 9). La mayoría de las cepas provino de hemocultivos (n: 72), seguida de cultivo de catéteres (n: 10) y líquidos peritoneales (n: 7) entre otros (n: 11).

Todas las cepas analizadas, presentaron CIM a anidulafungina con valores $\leq 2 \mu \mathrm{g} / \mathrm{mL}$. La concentración necesaria para inhibir el crecimiento de la mitad de las cepas estudiadas $\left(\mathrm{CIM}_{50}\right)$ fue de $0,125 \mu \mathrm{g} / \mathrm{mL}$ y para inhibir el 90\% de las cepas $\left(\mathrm{CIM}_{90}\right)$ correspondió a $1 \mu \mathrm{g} /$ $\mathrm{mL}$. Al analizar los datos por especie, observamos que las 33 cepas de $C$. albicans mostraron las menores CIM $_{50}$ y $\mathrm{CIM}_{90}$. Las demás especies de Candida spp, presentaron valores de $\mathrm{CIM}_{50}$ y de $\mathrm{CIM}_{90}$, en valores considerados sensibles, incluyendo a C. parapsilosis (Tabla 1).

Al comparar la interpretación de la susceptibilidad in vitro de las cepas estudiadas para anidulafungina, con los datos obtenidos previamente frente a fluconazol, se observa que las 16 cepas catalogadas como Sensible Dosis Dependiente (SDD), así como las ocho resistentes al azólico (4 C. krusei y 4 C. glabrata), presentaron CIM iguales o inferiores $0,5 \mu \mathrm{g} / \mathrm{mL}$ para esta equinocandina (Tabla 2).

*Nota del Editor: Nomenclatura reemplazada hoy en día por: enfermedad fúngica invasora. 


\begin{tabular}{|c|c|c|c|c|c|c|c|c|}
\hline & $\begin{array}{l}\text { C. albicans } \\
\text { (n: 33) }\end{array}$ & $\begin{array}{c}\text { C. parapsilosis } \\
\text { (n: 26) }\end{array}$ & $\begin{array}{l}\text { C. glabrata } \\
\text { (n: 19) }\end{array}$ & $\begin{array}{l}\text { C. Iusitaniae } \\
\text { (n: } 5)\end{array}$ & $\begin{array}{l}\text { C. tropicalis } \\
\qquad(\mathrm{n}: 9)\end{array}$ & $\begin{array}{l}\text { C. krusei } \\
(\mathrm{n}: 4)\end{array}$ & $\begin{array}{l}\text { otras* } \\
(n: 4)\end{array}$ & $\begin{array}{l}\text { Todas } \\
(\mathrm{n}: 100)\end{array}$ \\
\hline Rango & $\leq 0,03-2$ & $0,5-2$ & $0,0625-0,5$ & $0,0625-0,25$ & $0,0625-0,25$ & $0,25-0,5$ & $0,25-2$ & $<0,03-2$ \\
\hline $\mathrm{CIM}_{50}$ & 0,0625 & 1 & 0,125 & 0,25 & 0,125 & 0,25 & 0,25 & 0,125 \\
\hline $\mathrm{CIM}_{90}$ & 0,0625 & 2 & 0,25 & 0,25 & 0,25 & 0,5 & 2 & 1 \\
\hline
\end{tabular}

\begin{tabular}{|c|c|c|c|c|c|c|c|c|c|c|}
\hline \multirow[t]{2}{*}{ Cepa } & \multirow{2}{*}{$\begin{array}{l}\text { Interpretación } \\
\text { Fluconazol }\end{array}$} & \multicolumn{9}{|c|}{ CIM de anidulafungina $(\mu \mathrm{g} / \mathrm{mL})^{* *}$} \\
\hline & & $\leq 0,031$ & 0,0313 & 0,0625 & 0,125 & 0,25 & 0,5 & 1 & 2 & Total \\
\hline \multirow[t]{3}{*}{ C. albicans } & s & 4 & 2 & 22 & 1 & 2 & - & - & 1 & 32 \\
\hline & SDD & - & - & - & 1 & - & - & - & - & 1 \\
\hline & R & - & - & - & - & - & - & - & - & - \\
\hline \multirow[t]{3}{*}{ C. glabrata } & s & - & - & - & - & 1 & - & - & - & 1 \\
\hline & SDD & - & - & 3 & 7 & 3 & 1 & - & - & 14 \\
\hline & $R$ & - & - & - & 2 & 2 & - & - & - & 4 \\
\hline \multirow[t]{3}{*}{ C. parapsilosis } & s & - & - & - & - & - & 11 & 10 & 5 & 26 \\
\hline & SDD & - & - & - & - & - & - & - & - & - \\
\hline & $R$ & - & - & - & - & - & - & - & - & - \\
\hline \multirow[t]{3}{*}{ C. krusei } & s & - & - & - & - & - & - & - & - & - \\
\hline & SDD & - & - & - & - & - & - & - & - & - \\
\hline & R & - & - & - & - & 2 & 2 & - & - & 4 \\
\hline \multirow[t]{3}{*}{ C. tropicalis } & S & - & - & 4 & 3 & 2 & - & - & - & 9 \\
\hline & SDD & - & - & - & - & - & - & - & - & - \\
\hline & $R$ & - & - & - & - & - & - & - & - & - \\
\hline \multirow[t]{3}{*}{ C. Iusitaniae } & s & - & - & 2 & - & 3 & - & - & - & 5 \\
\hline & SDD & - & - & - & - & - & - & - & - & - \\
\hline & $\mathrm{R}$ & - & - & - & - & - & - & - & - & - \\
\hline \multirow[t]{3}{*}{ Otras* } & S & - & - & - & - & 1 & 1 & - & 1 & 3 \\
\hline & SDD & - & - & - & - & 1 & - & - & - & 1 \\
\hline & $\mathrm{R}$ & - & - & - & - & - & - & - & - & - \\
\hline
\end{tabular}

\section{Discusión}

Las levaduras del género Candida, son importantes agentes de infecciones oportunistas, principalmente en pacientes inmunodeprimidos o portadores de factores predisponentes, transformándose hoy en actores frecuentes de morbi-mortalidad, asociándose un cambio continuo y particular a cada región geográfica o tipo de hospital con la etiología y patrón de susceptibilidad ${ }^{2,3,7,10}$, en coincidencia con un aumento gradual de la resistencia, principalmente en cepas de Candida no-albicans ${ }^{4-6,16}$.
En estudios previos en nuestro país, se ha identificado a C. albicans como la especie prevalente en todo tipo de candidiasis, incluyendo EFI donde se ha reportado en aproximadamente la mitad de las candidemias ${ }^{7,8}$ ha prevalecido el serotipo $\mathrm{A}$ en adultos distribuyéndose homogéneamente los serotipos A y B en niños ${ }^{19}$. En este estudio, C. albicans correspondió a 33\% de las cepas estudiadas, con el objetivo de poder disponer de un mayor número de cepas de las especies de C. no-albicans, las cuales han mostrado una mayor relación con resistencia antifúngica ${ }^{2,4-7,16}$. 
El estudio de la susceptibilidad in vitro a anidulafungina evidenció $100 \%$ de sensibilidad, resultado comparable con un estudio de Pfaller y cols (2008), donde se analizaron 5.346 cepas con $98,8 \%$ de sensibilidad. Del total de cepas analizadas, la $\mathrm{CIM}_{50}$ y la $\mathrm{CIM}_{90}$ reflejan in vitro un rango para uso terapéutico seguro, siendo comparable con el estudio previamente mencionado, donde los valores de $\mathrm{CIM}_{50}$ y CIM $_{90}$ fue de 0,06 y $2 \mu \mathrm{g} / \mathrm{mL}$, respectivamente ${ }^{17}$.

El análisis de CIM por especies de levaduras frente a anidulafungina, evidencia que $C$. albicans presenta los menores valores de $\mathrm{CIM}_{50}$ y $\mathrm{CIM}_{90}$. Aunque $C$. parapsilosis evidenció los mayores valores de $\mathrm{CIM}_{50}$ y $\mathrm{CIM}_{90}$ entre las distintas especies analizadas, éstos se encuentran en los niveles considerados sensibles para este fármaco. Este fenómeno, particular a ciertas especies de levaduras, se presenta en todas las equinocandinas y se debería a efectos biológicos particulares de esta especie en la técnica de dilución en caldo ${ }^{16,17,28,29}$.

Otro aspecto interesante de exponer es el patrón de sensibilidad observado en C. krusei (especie intrínsecamente resistente a fluconazol) y C. glabrata (especie que desarrolla resistencia secundaria a fluconazol), cuyas cepas presentaron $\mathrm{CIM} \leq 0,5 \mu \mathrm{g} / \mathrm{mL}$ frente a anidulafungina, demostrando ser una buena alternativa terapéutica frente a ambas especies, en concordancia con estudios previos $^{16,18,29}$. La mejor actividad antifúngica in vitro de anidulafungina observada en estas cepas puede ser explicada por su mecanismo de acción, el cual interviene en la síntesis del componente 1,3- $\beta$-D-glucano en la pared celular fúngica ${ }^{11,12}$; en cambio, fluconazol actúa interrumpiendo la síntesis de ergosterol en la membrana celular, específicamente inhibiendo la acción de la enzima $14 \alpha$-desmetilasa ${ }^{5,6}$. Esta complementariedad de sitios de acción podría respaldar la aplicación in vivo de asociaciones de antimicóticos en ciertas condiciones clínicas, como candidemias, candidiasis aguda de diseminación hematógena y candidiasis hepato-esplénicas, entre otras ${ }^{30}$.

El presente estudio es el primero en Chile en evaluar la susceptibilidad in vitro de levaduras de interés clínico frente a anidulafungina, demostrando $100 \%$ de sensibilidad in vitro a esta equinocandina, lo que confirma su efectiva acción frente a estos agentes, incluso sobre cepas resistentes a fluconazol. Las cepas incluidas fueron aisladas antes de disponer de anidulafungina en nuestro medio, por lo que sugerimos repetir este análisis con cepas aisladas post incorporación de este antifúngico al mercado chileno, para evaluar la evolución en su patrón de sensibilidad.

Agradecimientos. Se agradece a Pfizer ${ }^{\circledR}$ Chile por el aporte del antifúngico anidulafungina para este estudio.

\section{Resumen}

Los hongos, especialmente las levaduras, se han transformado en importantes patógenos oportunistas y algunos de ellos tienen o desarrollan resistencia a los antifúngicos. Para enfrentar esta problemática se han desarrollado nuevas moléculas, como las equinocandinas. Este trabajo evaluó la susceptibilidad in vitro a anidulafungina en levaduras obtenidas previo a la incorporación de este antifúngico en Chile. Para ello, se seleccionaron 100 cepas de diversas especies aisladas de enfermedad fúngica invasora durante los años 2007 y 2008 en Chile, a las cuales se les midió la susceptibilidad in vitro por microdilución en caldo para anidulafungina según CLSI. Todas las cepas fueron sensibles a anidulafungina con $\mathrm{CIM}_{50} \mathrm{y}$ $\mathrm{CIM}_{90}$ de $0,125 \mu \mathrm{g} / \mathrm{mL}$ y $1 \mu \mathrm{g} / \mathrm{mL}$, respectivamente. Se detectó un ligero aumento de las CIM en C. parapsilosis respecto a las otras levaduras $\left(\mathrm{CIM}_{50}\right.$ de $1 \mu \mathrm{g} / \mathrm{mL} \mathrm{y} \mathrm{CIM}_{90}$ de $2 \mu \mathrm{g} / \mathrm{mL}$ ) considerándose estos valores en el rango de sensibilidad. La correlación de la susceptibilidad frente a fluconazol evidenció que cepas resistentes (n: 8) y sensibles dosis dependientes (n: 16) fueron sensibles a anidulafungina. Esta equinocandina aparece, in vitro, como una alternativa terapeutica efectiva frente a las levaduras aisladas en nuestros pacientes.

\section{Referencias}

1.- Calderone R A. Candida and Candidiasis. Editorial ASM Press, Washington DC; 2002. p. 37-53.

2.- Pfaller M A, Diekema D J. Epidemiology of invasive candidiasis: a persistent public health problem. Clin Microbiol Rev 2007; 20: 133-63.

3.- Richardson M, Lass-Flörl C. Changing epidemiology of systemic fungal infections. Clin Microbiol Infect 2008; 14 Suppl 4: 5-24.

4.- Martínez-Suárez J V, Rodríguez-Tudela J L. La resistencia a antifúngicos en los hongos patógenos oportunistas (II). Imidazoles y triazoles. Enferm Infecc Microbiol Clin 1996; 14: 490-8.
5.- Marr K A, Bowden R A. Clinical, cellular, and molecular factors that contribute to antifungal drug resistance. Clin Microbiol Rev 1998; 11: 382-402.

6.- Ghannoum M A, Rice L B. Antifungal agents: mode of action, mechanisms of resistance, and correlation of these mechanisms with bacterial resistance. Clin Microbiol Rev 1999; 12: 50117

7.- Silva V, Díaz M C, Febré N, and Chilean Invasive Fungal Infection Group. Invasive fungal infections in Chile: a multicenter study of fungal prevalence and susceptibility during a 1-year period. Med Mycol 2004; 42: 333-9.

8.- Silva V, Alvarado D, Díaz M C. Antifungal susceptibility of 50 Candida isolated from invasive mycosis in Chile. Med Mycol 2004; 42: 283-5.

9.- Alburquerque C, Hermosilla G, Tapia C. Distribución y susceptibilidad a fluconazol de levaduras del género Candida aisladas en pacientes hospitalizados y ambulatorios. Rev Chil Infectol 2009; 26: 435-9.

10.- Pfaller M A, Jones R N, Doern G, Sader H S, Hollis R J, for the Sentry Participant Group. International surveillance of bloodstream infections due to Candida species: frequency of occurrence and antifungal susceptibilities of isolates collected in 1997 in the United States, Canada, and South America for the 
SENTRY Program. J Clin Microbiol 1998; 36 : 1886-9.

11.- Perlin, D. S. Resistance to echinocandin-class antifungal drugs. Drug Resist Updat 2007; 10: 121-30.

12.- Rogers T R, Johnson E M, Munro C. Echinocandin antifungal drug resistance. J Invasive Fungal Infect 2007; 1: 99-105.

13.- Nelson P W, Lozano-Chiu M, Rex J H. In vitro growth inhibitory activity of pneumocandins L-733, 569 and L-743m 872 against putatively amphotericin B- and fluconazole-resistant Candida isolates: influence of assay conditions. J Med Vet Mycol 1997; 35: 285-7.

14.- Bennett J E. Echinocandins for candidemia in adults without neutropenia. N Engl J Med 2006; 355: 1154-9.

15.- Espinel-Ingroff A. In vitro antifungal activities of anidulafungin and micafungin, licensed agents and the investigational triazole posaconazole as determined by NCCLS methods for 12,052 fungal isolates: review of the literature. Rev Iberoam Micol 2003; 20 : 121-36.

16.- Pfaller M A, Boyken L, Hollis R J, Messer S A, Tendolkar S, Diekema D J. In vitro activities of anidulafungin against more than 2,500 clinical isolates of Candida spp., including 315 isolates resistant to fluconazole. J Clin Microbiol 2005; 43: 5425-7.

17.- Pfaller M A, Boyken L, Hollis R J, Kroeger J, Messer S A, Tendolkar S, et al. In vitro susceptibility of invasive isolates of Candida spp. to anidulafungin, caspofungin, and micafungin: six years of global surveillance. J Clin Microbiol 2008; 46: 150-6.

18.- Reboli A C, Rotstein C, Pappas P G, Chapman S W, Kett D H, Kumar D, et al, Anidulafungin Study Group. Anidulafungin versus fluconazole for invasive candidiasis. N Engl J Med 2007; 356: 2472-82.

19.- Silva V, Cabrera M, Díaz MC, Abarca C, Hermosilla G. Prevalencia de serotipos de Candida albicans en aislamientos de hemocultivo en Chile y primer caso de candidemia por Candida dubliniensis. Rev Iberoam Micol 2003; 20: 46- 51.

20.- Ramani R, Gromadzki S, Pincus D H, Salkin I F, Chaturvedi. Efficacy of API 20C and ID 32C systems for identification of common and rare clinical yeasts isolates. J Clin Microbiol 1998; 36: 3396-8.

21.- National Committee for Clinical Laboratory Standards. Methods for antifungal disk diffusion susceptibility testing of yeasts: approved standard M44-A. NCCLS, Wayne, PA. USA, 2004.

22.- Arikan S. Current status of antifungal susceptibility testing methods. Med Mycol 2007; 45: 569-87.

23.- Tapia C. Actualización en pruebas de susceptibilidad antifúngica. Rev Chil Infectol 2009; 26: 144-50.

24.- Rodero L, Córdova S, Vivot W, Campo M, Corfield P, Olguín C, et al. Método de difusión con discos para la determinación de sensibilidad a fluconazol en aislamientos de Candida spp. Rev Arg Microbiol 2006; 38: 155-63.

25.- Negri M, Henriques M, Svidzinski T, Rodrigues C, Oliveira R. Correlation between E-tests, disk diffusion, and microdilution methods for antifungal susceptibility testing of Candida species from infection and colonization. J Clin Labo Anal 2009; 23: 324-30.

26.- National Committee for Clinical Laboratory Standards. Reference method for broth dilution antifungal susceptibility testing of yeasts; approved standard NCCLS document M27-A2. Wayne, PA: National Committee for Clinical Laboratory Standard. 2002.

27.- Clinical Laboratory Standards Institute. Quality control minimal inhibitory concentration (MIC) for broth microdilution and MIC intrepretative breakpoint (M27-S2). 2006.

28.- Bartizal C, Odds F C. Influences of methodological variables on susceptibility testing of caspofungin against Candida species and Aspergillus fumigatus. Antimicrob Agents Chemother 2003; 47: 2100-7.

29.- Pfaller M A, Boyken L, Hollis J, Messer A, Tendolkar S, Diekema D. In vitro susceptibilities of Candida spp. to caspofungin: four years of global surveillance. J Clin Microbiol 2006; 44 : 760-763

30.- Pappas P, Rex JH, Sobel J, Filler S, Dismukes W, Walsh TJ, et al. Guidelines for treatment of candidiasis. Clin Infect Dis 2004; 38: 161- 89 . 